\title{
Clinical efficacy of sevelamer hydrochloride in patients with end-stage renal disease: a retrospective study
}

\author{
Sartaj Alam $^{1,2}$, MCPS, FCPS, Asrar $\underline{\text { Hussain }}^{2}$, FCPS, Rajendra Daiwajna², MRCP, Jackson Tan $^{2}$, FRCP
}

INTRODUCTION Sevelamer hydrochloride (Renagel) is frequently used as a second-line phosphate binder in patients on renal replacement therapy. Many studies have shown that sevelamer can improve vascular calcification, serum uric acid and low-density lipoprotein (LDL) cholesterol levels. The main objectives of this study were to assess the efficacy of sevelamer against calcium-based phosphate binders, as well as its tolerability and side-effect profile.

METHODS This was a retrospective study that included all patients on renal replacement therapy (between 2008 and 2011) who had previously received calcium-based binders for $\geq 6$ months and were subsequently switched to sevelamer. Data collected from the patients' medical records included demographics, as well as renal parameters three months prior to sevelamer treatment, and at three and six months post treatment. The study excluded patients on multiple, concomitant phosphate binders or with functioning renal transplants, and those who were noncompliant or had inadequate follow-up blood investigations.

RESULTS A total of 39 patients were included in the study. No major side effects were reported by any of the patients. There were improvements in calcium, phosphate, uric acid and LDL cholesterol levels at three and six months postsevelamer treatment.

CONCLUSION We found sevelamer to be superior to calcium-based phosphate binders in reducing serum calcium, phosphate, uric acid and LDL cholesterol levels in our patient population with advanced renal bone disease. Sevelamer also appears to be well tolerated with no significant side effects.

Keywords: calcium-based binders, dialysis, phosphate binders, renagel, sevelamer

\section{INTRODUCTION}

Strongly linked to secondary hyperparathyroidism, renal osteodystrophy, and vascular and soft tissue calcification, hyperphosphataemia if left uncontrolled can lead to increased cardiovascular mortality in patients with end-stage renal disease. According to some studies, adjusted mortality increases by $20 \%-40 \%$ with increases in serum phosphate levels (up to $4.2 \mathrm{mmol} / \mathrm{L}){ }^{(1,2)}$ Calcium levels are also commonly raised as a result of hyperparathyroidism, and this together with hyperphosphataemia can increase the calcium-phosphate product, which is an independent factor for cardiac mortality. ${ }^{(3)}$

Calcium-based phosphate binders have been used in medical practice for many years, although they can increase serum calcium levels and therefore predispose patients to vascular and cardiac calcifications. ${ }^{(4)}$ Likewise, although aluminium-containing agents are highly effective, they are related to accumulation and toxicity. ${ }^{(5)}$ Lanthanum carbonate is an alternative non-calciumbased binder that has promising phosphate-binding effects. However, long-term data on the safety and coronary calcification of lanthanum are still under debate.(6) Sevelamer hydrochloride (Renagel) is non-metal-based and frequently used as a secondline phosphate binder in patients on renal replacement therapy. Several randomised controlled studies have shown that sevelamer can reduce serum calcium levels and the incidence of hypercalcaemia. ${ }^{(7-9)}$ These studies have also shown that sevelamer is equipotent to calcium-based phosphate binders in reducing serum phosphate levels. Although many small studies have confirmed sevelamer's effects on slowing coronary calcifications in both dialysis and predialysis patients, there are still no long-term data that support the use of sevelamer over other phosphate-binding agents in the reduction of mortality. ${ }^{(10,11)}$ According to some authors, treatment with sevelamer can also reduce all-cause hospitalisations as compared to treatment with calcium-based binders. ${ }^{(12)}$

In addition to the phosphate-reducing and calcium-sparing properties of sevelamar, many small studies have shown that its use is linked to improvements in serum uric acid, low-density lipoprotein (LDL) cholesterol and C-reactive protein levels. ${ }^{(13-15)} \mathrm{As}$ sevelamer is not absorbed by the gastrointestinal tract, it has a relatively low incidence of side effects. It also has the ability to bind and sequester bile acid, which may explain its lipid-lowering effect. ${ }^{(4)}$ It has also been postulated that sevelamer decreases serum uric acid concentration in maintenance haemodialysis patients through the adsorption of uric acid. ${ }^{(16)}$ However, much like lanthanum, the widespread appeal of sevelamer is hampered by its relatively high cost, which thus limits its use in clinical settings. ${ }^{(6)}$ A literature search of all PubMed-listed Asian studies on sevelamer revealed that it is not commonly used in Asian

${ }^{1}$ Renal unit, Suri Seri Begawan Hospital, Kuala Belait Dialysis Centre, Kuala Belait, ${ }^{2}$ Department of Renal Medicine, Raja Isteri Pengiran Anak Saleha Hospital, Bandar Seri Begawan, Brunei Darussalam

Correspondence: Dr Sartaj Alam, Renal Physician, Renal Unit, Suri Seri Begawan Hospital, Kuala Belait Dialysis Center, Suri Seri Begawan Hospital, Kuala Belait, KB 2133, Brunei Darussalam. drsartajalam@hotmail.com 
countries, probably due to its high cost and differences in regional marketing policies.

The main objectives of this study were to assess: (a) the efficacy of sevelamer against calcium-based phosphate binders, in terms of its effects on serum phosphate, calcium, parathyroid hormone, bicarbonate, uric acid and lipid levels; and (b) its tolerability and side-effect profile. Our study aimed to provide new information on the efficacy and tolerability of sevelamer in a dialysis population predominantly composed of patients of Malay ethnicity.

\section{METHODS}

This was a retrospective study that assessed the effects of sevelamer in patients on renal replacement therapy who had previously received calcium-based phosphate binders for $\geq 6$ months. In our hospital, patients are usually switched from calcium-based binders to sevelamer if they exhibit features of tertiary hyperparathyroidism (especially hypercalcaemia), uncontrolled hyperphosphataemia or intolerance to calciumbased binders. The study included all patients on renal replacement therapy between 2008 and 2011 who were on calcium-based phosphate binders for $\geq 1$ year and then switched to sevelamer treatment. The study excluded patients on multiple, concomitant phosphate binders or with functioning renal transplants, and those who were noncompliant or had inadequate follow-up blood investigations.

Data were retrospectively collected from the patients' medical records, and included general demographic details, comorbidities, dialysis modality and duration, and serum levels of calcium, phosphate, parathyroid hormone, bicarbonate, uric acid and total cholesterol. Patients were also interviewed during routine clinical checks with regard to any side effects experienced or compliance issues while undergoing sevelamer treatment. Target renal parameters at three months pre-sevelamer treatment (or at baseline if patients were on calcium-based phosphate binders), and at three and six months post-sevelamer treatment were compared. Differences between pairs were calculated using Student's $t$-test. Two-tailed $p<0.05$ was considered statistically significant.

\section{RESULTS}

A total of 39 patients were included in the study. The mean age of the patients was $47.90 \pm 14.61$ years (median 46 years) and there was a male preponderance of $51.28 \%$. Comorbidities observed among patients included hypertension (89.74\%), diabetes mellitus (20.51\%), ischaemic heart disease (17.95\%) and parathyroidectomy $(12.82 \%)$. The demographic characteristics of the patients are summarised in Table I. No serious side effects were reported by any of the patients.

The main reasons that prompted the switch from calciumbased phosphate binders to sevelamer included hypercalcaemia $(\mathrm{n}=15,38.5 \%)$, inadequate phosphate control $(\mathrm{n}=8,20.5 \%)$, and intolerance to calcium-based binders $(n=2,5.1 \%)$. The
Table I. Demographic characteristics of patients $(n=39)$.

\begin{tabular}{lc}
\hline Characteristics & No. (\%) \\
\hline Male gender & $20(51.28)$ \\
Mean \pm SD; median age (yrs) & $47.90 \pm 14.61 ; 46.00$ \\
Malay ethnicity & $37(94.87)$ \\
Dialysis & \\
Haemodialysis & $32(82.05)$ \\
Peritoneal dialysis & $7(17.95)$ \\
Mean duration of dialysis \pm SD (yrs) & $6.70 \pm 4.82$ \\
Comorbidity & \\
Hypertension & $35(89.74)$ \\
Diabetes mellitus & $8(20.51)$ \\
Ischaemic heart disease & $7(17.95)$ \\
Parathyroidectomy & $5(12.82)$ \\
\hline SD: standard deviation &
\end{tabular}

reason for the switch was unknown for 14 (35.9\%) patients, as it was not documented. The serum levels of various biochemical parameters at three months pre-sevelamer treatment, and at three and six months post-sevelamer treatment, are presented in Table II. There was significant improvement in the calcium, phosphate, uric acid and LDL cholesterol levels of the patients at three and six months post-sevelamer treatment. Table III presents the number of patients in whom reduced, unchanged or increased biochemical parameters were seen at three and six months post-sevelamer treatment, respectively.

\section{DISCUSSION}

A search of the published literature listed in PubMed's database revealed that few studies had reported on the efficacy of sevelamer in Asian patients of non-Japanese descent. A summary of studies on sevelamer from Taiwan, Hong Kong, India and Saudi Arabia is presented in Table IV. ${ }^{(17-22)}$ A majority of these studies compared the efficacy of calcium-based binders and sevelamer with regard to bone turnover. The consensus is that sevelamer is as good as calcium-based binders in reducing phosphate, but superior to calcium-based binders in reducing hypercalcaemia. Studies from Hong Kong have shown that sevelamer was cost-effective at lower doses and effective in patients with severe hyperphosphataemia. ${ }^{(19,20)}$

Our study is thus the first of its kind reporting the efficacy of sevelamer treatment in an Asian population composed primarily of patients of Malay ethnicity on renal replacement therapy. Similar to other Asian studies, we also found a reduction in calcium and calcium-phosphate products following sevelamer treatment when compared to treatment with calcium-based phosphate binders. Compared with calcium-based binders, sevelamer also appeared to have significantly better phosphate-reducing properties. Our results are particularly significant, as many of our patients already had advanced renal bone disease with high serum calcium and phosphate levels that were unresponsive to therapy with calcium-containing binders. A trend toward better lipid and uric acid control was also observed in patients after sevelamer treatment. Expectedly, due to its hydrochloride content, the use of sevelamer was associated with lower serum 
Table II. Biochemical parameter levels pre- and post-sevelamer treatment.

\begin{tabular}{|c|c|c|c|c|c|}
\hline \multirow[t]{2}{*}{ Serum parameter* } & \multirow{2}{*}{$\begin{array}{l}\text { Pretreatment } \\
\quad \text { level }^{+}\end{array}$} & \multicolumn{2}{|c|}{ At $3 \mathrm{mths}$ posttreatment } & \multicolumn{2}{|c|}{ At 6 mths posttreatment } \\
\hline & & Level & p-value & Level & p-value \\
\hline Calcium (mmol/L) & $2.54 \pm 0.25$ & $2.41 \pm 0.24$ & $<0.05$ & $2.44 \pm 0.27$ & $<0.05$ \\
\hline Phosphate (mmol/L) & $2.58 \pm 0.65$ & $2.18 \pm 0.46$ & $<0.05$ & $2.10 \pm 0.49$ & $<0.05$ \\
\hline Bicarbonate (mmol/L) & $22.35 \pm 3.30$ & $21.33 \pm 4.04$ & 0.1210 & $19.79 \pm 3.57$ & $<0.05$ \\
\hline Total cholesterol (mmol/L) & $4.78 \pm 1.26$ & $4.11 \pm 1.23$ & $<0.05$ & $3.95 \pm 1.14$ & $<0.05$ \\
\hline LDL cholesterol (mmol/L) & $2.84 \pm 1.03$ & $2.40 \pm 1.06$ & $<0.05$ & $2.29 \pm 0.96$ & $<0.05$ \\
\hline Uric acid $(\mu \mathrm{mol} / \mathrm{L})$ & $414.14 \pm 84.73$ & $379.71 \pm 69.26$ & $<0.05$ & $372.55 \pm 69.25$ & $<0.05$ \\
\hline Parathyroid hormone (pmol/L) & $94.33 \pm 65.11$ & $103.67 \pm 74.77$ & 0.0763 & $108.77 \pm 99.05$ & 0.1050 \\
\hline
\end{tabular}

${ }^{*}$ Data is expressed as mean \pm standard deviation. ${ }^{\dagger}$ Patients were on calcium-based binders.

LDL: low-density lipoprotein

Table III. Frequency of the changes in patients' biochemical parameters at 3 and 6 months post-sevelamer treatment $(n=39)$.

\begin{tabular}{|c|c|c|c|c|c|c|}
\hline \multirow[t]{2}{*}{ Serum parameter } & \multicolumn{3}{|c|}{ At $3 \mathrm{mths}$ posttreatment } & \multicolumn{3}{|c|}{ At $6 \mathrm{mths}$ posttreatment } \\
\hline & Reduced & Unchanged & Increased & Reduced & Unchanged & Increased \\
\hline Calcium & 8 & 31 & 0 & 7 & 30 & 2 \\
\hline Phosphate & 16 & 20 & 3 & 14 & 20 & 5 \\
\hline Bicarbonate & 8 & 28 & 3 & 11 & 23 & 5 \\
\hline Total cholesterol & 11 & 26 & 2 & 10 & 26 & 3 \\
\hline LDL cholesterol & 13 & 22 & 4 & 14 & 22 & 3 \\
\hline Uric acid & 6 & 30 & 3 & 9 & 22 & 8 \\
\hline Parathyroid hormone & 11 & 13 & 15 & 9 & 14 & 16 \\
\hline
\end{tabular}

'Reduced' is defined as $>20 \%$ reduction from baseline value. 'Increased' is defined as $<20 \%$ increment from baseline value.

LDL: low-density lipoprotein

Table IV. Summary of Asian studies on sevelamer in the literature.

\begin{tabular}{|c|c|c|c|c|c|}
\hline Study (year) & Country & $\begin{array}{l}\text { No. of } \\
\text { patients }\end{array}$ & Study design & $\begin{array}{l}\text { Patient } \\
\text { type }\end{array}$ & Study objective \\
\hline Shaheen et al (2004) $)^{(17)}$ & Saudi Arabia & 12 & $\begin{array}{l}\text { Randomised, open-label, } \\
\text { cross-over }\end{array}$ & $H D$ & Comparison with calcium-based binders \\
\hline Lieu et al $(2006)^{(18)}$ & Taiwan & 37 & Randomised, open-label & $H D$ & Comparison with calcium-based binders \\
\hline Chow et al (2007)(19) & Hong Kong & 27 & Randomised, open-label & PD & Low dose vs. high dose \\
\hline Lo et al $(2008)^{(20)}$ & Hong Kong & 20 & Open-label & PD & $\begin{array}{l}\text { Assess side effects and efficacy in } \\
\text { severe hyperphosphataemia }\end{array}$ \\
\hline Gulati et al (2010)(21) & India & 22 & Randomised, open-label & CKD 3-4 & Comparison with calcium-based binders \\
\hline Lin et al $(2010)^{(22)}$ & Taiwan & 26 & Randomised, open-label & $H D$ & Comparison with calcium-based binders \\
\hline
\end{tabular}

CKD 3-4: chronic kidney disease stages 3-4; HD: haemodialysis; PD: peritoneal dialysis

bicarbonate levels. However, this effect was only evident after six months of sevelamer treatment. The authors are of the view that secondary and tertiary hyperparathyroidism would be better controlled with reductions in serum calcium and phosphate levels via an incremental use of vitamin D derivatives. However, as the present study was performed retrospectively, we were unable to reliably measure changes in vitamin $\mathrm{D}$ or calcimimetic doses before and after sevelamer treatment. Therefore, one would logically expect an increase in parathyroid hormone levels at follow-up six months post-sevelamer treatment due to the expected progression of renal bone disease. However, judging by the stable parathyroid hormone levels seen at six months post-sevelamer treatment in our cohort, we speculate that the low calcium and phosphate levels observed following six months of sevelamer treatment had enabled more vitamin D to be used, thus maintaining a stable parathyroid status.
No major side effects were reported by our patients, with only three reporting mild gastrointestinal disturbances, although many patients reported that sevelamer tablets were more difficult to swallow, as they were bigger than calcium-based binders. Despite this, the inconvenience caused was not severe enough for any of our patients to discontinue medication or revert to calcium-based binders. However, it is also possible that some patients may not have been entirely truthful about the drug's side effects. This can be attributed to the conservative, noncritical nature of Brunein culture, which might have led our cohort to be reluctant to reveal all the side effects experienced. Some participants might have also been ignorant of the medication they were prescribed and the accompanying side effects. Given these factors, a prospective trial involving a placebo group or with a crossover design might have been better for ascertaining the true side effects of the medication. 
We acknowledge that there are limitations to this study. Most of our patients who were started on sevelamer already had advanced metabolic bone disorder, with high serum calcium, phosphate and parathyroid hormone levels. It is likely that the withdrawal of calcium-based binders in these patients (who were mostly secondary and tertiary hyperparathyroid patients) would have invariably led to a reduction in calcium levels regardless of the use of sevelamer. We were unable to justify using a crossover trial for these patients, as it would be unethical to discontinue sevelamer in patients with 'corrected' bone biochemistry, as many would have already tried the calcium-based formulations and found them to be ineffective.

We conclude that sevelamer is effective as a second-line agent in the control of hypercalcaemia and hyperphosphataemia in patients with advanced renal bone disease. Sevelamer may also play a role in delaying parathyroidectomy, as it allows for the incremental use of vitamin D derivatives to control tertiary hyperparathyroidism. We also observed a trend toward better lipid and uric acid control in patients receiving sevelamer. Finally, we opine that more studies are needed in order to examine the effects of sevelamer as a first-line phosphate binder in patients with early renal bone disease.

\section{REFERENCES}

1. Melamed ML, Eustace JA, Plantinga L, et al. Changes in serum calcium, phosphate, and PTH and the risk of death in incident dialysis patients: A longitudinal study. Kidney Int 2006; 70:351-7.

2. Block GA, Port FK. Re-evaluation of risk associated with hyperphosphatemia and hyperparathyroidism in dialysis patients: Recommendations for a change in management. Am J Kidney Dis 2000; 35:1226-37.

3. Kuhlmann MK. Management of hyperphosphatemia. Hemodial Int 2006; 10:338-45.

4. Amin N. The impact of improved phosphorus control: use of sevelamer hydrochloride in patients with chronic renal failure. Nephrol Dial Transplant 2002; 17:340-5.

5. Mudge DW Johnson DW, Hawley CM, etal. Doaluminium-based phosphate binders continue to have a role in contemporary nephrology practice? BMC Nephrol 2011; 12:20.

6. Hutchinson AJ, Smith CP, Brenchley PE. Pharmacology, efficacy and safety of oral phosphate binders. Nat Rev Nephrol 2011; 7:578-89.

7. Chertow GM, Burke SK, Raggi P, Treat to Goal Working Group. Sevelamer attenuates the progression of coronary and aortic calcification in hemodialysis patients. Kidney Int 2002; 62:245-52.
8. Qunibi W Moustafa M, Muenz LR, et al. A 1 year randomised trial of calcium acetate versus sevelamer on progression of coronary artery calcification in haemodialysis patients with comparable lipid control: the Calcium Acetate Renagel Evaluation-2 (CARE-2) study. Am J Kidney Dis 2008; 51:952-65.

9. Block GA, Spiegel DM, Ehrlich J, et al. Effects of sevelamer and calcium on coronary artery calcification in patients new to hemodialysis. Kidney Int 2005; 68:1815-24.

10. Block GA, Raggi P, Bellasi A ,Kooienga L, Spiegel DM. Mortality effect of coronary calcification and phosphate binder choice in incident hemodialysis patients. Kidney Int 2007; 71:438-41.

11. Russo D, Miranda I, Ruocco C, et al. The progression of coronary artery calcification in predialysis patients on calcium carbonate or sevelamer. Kidney Int 2007; 72:1255-61.

12. St Peter WL, Liu J, Weinhandl E, Fan Q. A comparison of sevelamer and calcium-based phosphate binders on mortality, hospitalization, and morbidity in hemodialysis: a secondary analysis of the Dialysis Clinical Outcomes Revisited (DCOR) randomized trial using claims data. Am J Kidney Dis 2008; 51:445-54.

13. Garg JP, Chasan-Taber S, Blair A, et al. Effects of sevelamer and calciumbased phosphate binders on uric acid concentrations in patients undergoing hemodialysis: A randomized clinical trial. Arthritis Rheum 2005; 52:290-5.

14. Shantouf R, Budoff MJ, Ahmadi N, et al. Effects of sevelamer and calcium-based phosphate binders on lipid and inflammatory markers in hemodialysis patients. Am J Nephrol 2008; 28:275-9.

15. Ferramosca E, Burke S, Chasan-Taber S, et al.: Potential antiatherogenic and anti-inflammatory properties of sevelamer in maintenance hemodialysis patients. Am Heart J 2005; 149:820-5.

16. Ohno I, Yamaguchi Y, Saikawa H, et al. Sevelamer decreases serum uric acid concentration through adsorption of uric acid in maintenance hemodialysis patients. Intern Med 2009; 48:415-20.

17. Shaheen FA, Akeel NM, Badawi LS, Souqiyyeh MZ. Efficacy and safety of sevelamer. Comparison with calcium carbonate in the treatment of hyperphosphatemia in hemodialysis patients. Saudi Med J 2004; 25:785-91.

18. Lieu YL, Lin HH, Yu CC, et al. A comparison of sevelamer hydrochloride with calcium acetate on biomarkers of bone turnover in hemodialysis patients. Ren Fail 2006; 28:701-7.

19. Chow KM, Szeto CC, Kwan BC, Leung CB, Li PK. Sevelamer treatment strategy in peritoneal dialysis patients: conventional dose does not make best use of resources. J Nephrol 2007; 20:674-82.

20. Lo WK, Cheng SW, Ng SY, et al. Efficacy and side effects of sevelamer hydrochloride as sole phosphate binder in peritoneal dialysis patients with severe hyperphosphatemia. Perit Dial Int 2008; 28:93-5.

21. Gulati A, Sridhar V, Bose T, Hari P, Bagga A. Short-term efficacy of sevelamer versus calcium acetate in patients with chronic kidney disease stage 3-4. Int Urol Nephrol 2010; 42:1055-62.

22. Lin YF, Chen YM, Hung KY, et al. Benefits of sevelamer on markers of bone turnover in Taiwanese hemodialysis patients. J Formos Med Assoc 2010; 109:663-72. 